\title{
Short-Term Effects of Non-Surgical Periodontal Treatment on the Gingival Crevicular Fluid Cytokine Profiles in Sites With Induced Periodontal Defects: A Study on Dogs With and Without Streptozotocin-Induced Diabetes
}

Fawad Javed, * Nasser M. Al-Daghri, ${ }^{\dagger}$ Hom-Lay Wang, ${ }^{\ddagger}$ Cun Yu Wang,,$§$ and Khalid Al-Hezaimi*\|

Background: The aim of this study is to assess the short-term effects of non-surgical periodontal therapy (NSPT) on the gingival crevicular fluid (GCF) cytokine profile in sites with standardized periodontal bony defects in beagle dogs with and without diabetes.

Methods: Four beagle dogs with streptozotocin (STZ)-induced diabetes and four healthy dogs were included. Fasting blood glucose levels were measured using a glucometer. In all animals, a 3-walled bony defect was created on the mesial surface of the second premolar and first molar in all quadrants. After 12 weeks, all animals underwent weekly NSPT for 3 weeks. Baseline and post-NSPT GCF samples were collected, and levels of interleukin (IL)-1, IL-1 $\beta$, IL-6, IL-8, and tumor necrosis factor (TNF)- $\alpha$ were measured using enzyme-linked immunosorbent assay. Statistical analyses were performed using a software program, and $P$ values $<0.05$ were considered statistically significant.

Results: Mean fasting blood glucose levels were significantly higher in dogs with induced diabetes than those without diabetes $(P<0.01)$. At baseline, mean IL-6 $(P<0.01)$ and IL-8 $(P<0.05)$ levels were higher in dogs with diabetes than those without diabetes. A significant reduction in levels of IL-1, IL-1 $\beta$, IL-6, IL-8, and TNF- $\alpha$ was noted in dogs without diabetes 1 week after NSPT. However, this significant reduction $(P<0.05)$ only appeared 2 weeks after NSPT in dogs with diabetes.

Conclusions: NSPT reduces GCF levels of proinflammatory cytokines in dogs with and without STZinduced diabetes; however, chronic hyperglycemia seems to retard the effect of NSPT on GCF cytokine concentration. J Periodontol 2014;85:1589-1595.

\section{KEY WORDS}

Alveolar bone loss; cytokines; diabetes mellitus; periodontal debridement.

\footnotetext{
* Engineer Abdullah Bugshan Research Chair for Growth Factors and Bone Regeneration, 3D Imaging and Biomechanical Laboratory, College of Dentistry, King Saud University, Riyadh, Saudi Arabia.

$\dagger$ Department of Biochemistry, College of Science, King Saud University.

Department of Periodontics and Oral Medicine, School of Dentistry, University of Michigan, Ann Arbor, MI.

$\S$ Dr. No-Hee Park Endowed Chair in Dentistry and the Chair of the Division of Oral Biology and Medicine; University of California, Los Angeles; Los Angeles, CA.

\| Department of Periodontics and Community Dentistry, College of Dentistry, King Saud University.
} 
I $\mathrm{t}$ is well established that systemic conditions influence periodontal health. ${ }^{1-4}$ Studies ${ }^{4-8}$ reported that periodontal inflammatory conditions are worse in patients with poorly controlled diabetes compared with those with well-controlled diabetes and individuals without diabetes. Traditionally, severity of periodontal disease is measured via clinical and radiographic parameters (such as plaque index [PI], bleeding on probing [BOP], probing depth [PD], and marginal bone loss); $4,5,8$ however, laboratory-based techniques may also yield valuable information in this context.

The gingival sulcus contains an osmotically mediated inflammatory exudate, the gingival crevicular fluid (GCF), which increases in volume under periodontal inflammatory conditions (because of capillary permeability). Biochemical assessment of GCF is a non-invasive technique for assessing the host response in individuals with periodontitis. It is known that patients with periodontitis display higher concentrations of proinflammatory proteins, such as cytokines, in their GCF compared with individuals without periodontitis. Studies ${ }^{9-15}$ reported that patients with periodontitis, with and without diabetes, display higher concentrations of various cytokines, including interleukin (IL)-1 $\beta$, IL-6, matrix metalloproteinases 8 and 9 , and tumor necrosis factor (TNF)- $\alpha$ in the GCF. Increased expression of such proinflammatory cytokines in the GCF has been associated with the production of reactive oxygen species (ROS) produced as a result of oxidative stress under periodontal inflammatory conditions. ${ }^{16}$ In addition, an increased formation and accumulation of advanced glycation end products (AGEs) in the periodontal tissues under hyperglycemia has also been proposed to increase the burden of proinflammatory cytokines in the serum and GCF. ${ }^{17,18}$ In this context, analysis of proinflammatory cytokines in GCF provides valuable information regarding the pathophysiologic processes associated with periodontitis.

Non-surgical periodontal therapy (NSPT) (with and without adjunctive remedies) is a classic nonsurgical therapy for treating periodontitis. ${ }^{6,10,19,20}$ It was reported that NSPT is effective in reducing the levels of proinflammatory cytokines in GCF from patients with periodontitis with and without type 2 diabetes. ${ }^{10}$ In a recent literature review, Javed et al. ${ }^{9}$ reported that the GCF cytokine profile in patients with and without diabetes is governed by the intensity of periodontal inflammation, and the role of diabetes in this regard is rather secondary. Therefore, it was hypothesized that regular plaque control regimens in animals with standardized periodontal bony defects (representing a clinical scenario of adult chronic periodontitis [CP]) with and without diabetes would demonstrate a gradual yet significant reduction in the levels of proinflammatory cytokines in GCF.

The aim of the present experiment is to assess the short-term effects of NSPT on the GCF cytokine profile in sites with standardized periodontal bony defects in beagle dogs with and without streptozotocin (STZ)-induced diabetes.

\section{MATERIALS AND METHODS}

\section{Ethical Guidelines}

The study was approved by the research ethics review committee of the Engineer Abdullah Bugshan Research Chair for Growth Factors and Bone Regeneration, 3D Imaging and Biomechanical Laboratory, College of Applied Medical Sciences, King Saud University, Riyadh, Saudi Arabia.

\section{Study Animals}

Eight male beagle dogs (mean age: $12 \pm 0.4$ months; weight: $13 \pm 1.2 \mathrm{~kg}$ ) were used in this study. All animals were kept in individual cages throughout the study period.

All surgical and non-surgical procedures were performed under general anesthesia using ketamine (10 $\mathrm{mg} / \mathrm{kg}$ body weight) with buccal infiltration of xylocaine.\#

\section{Induction of Experimental Diabetes}

Four dogs were placed on a fasting regimen for 24 hours before the induction of diabetes via intravenous injection of STZ. Blood was collected for baseline levels of fasting plasma glucose. Because there is a risk of fatal hypoglycemia as a result of massive pancreatic insulin release, dogs were treated with $10 \%$ glucose solution after 4 hours of diabetes induction. ${ }^{21}$ After 5 days, the dogs showed fasting hyperglycemia ( $\geq 10 \mathrm{mmol} / \mathrm{L})$.

\section{Induction of Periodontal Bony Defects}

A 3-walled periodontal bony defect $(3 \times 5 \mathrm{~mm})$ involving the interdental bone was created on the mesial walls of the second premolars (P2) and first molars (M1) in all quadrants (Figs. 1 and 2). Intramuscular amoxicillin** (25 mg/kg body weight) was administered at the day of surgery. Plaque control regimens (NSPT and chlorhexidine rinses) were started 12 weeks after defect induction. All animals underwent weekly NSPT under general anesthesia using an ultrasonic scaler ${ }^{\dagger \dagger}$ for 3 weeks.

\section{Collection of GCF Samples and Enzyme-Linked Immunosorbent Assay}

GCF samples were collected at baseline (12 weeks after defect induction) and after weeks 1,2, and 3

\footnotetext{
II Pfizer Limited, Sandwich, Kent, U.K.

\# AstraZeneca LP for DENTSPLY Pharmaceutical, York, PA.

** Betamox LA, Norbrook Laboratories, Newry, County Down, Northern Ireland.

$\dagger \dagger$ Hu-Friedy, Chicago, IL.
} 


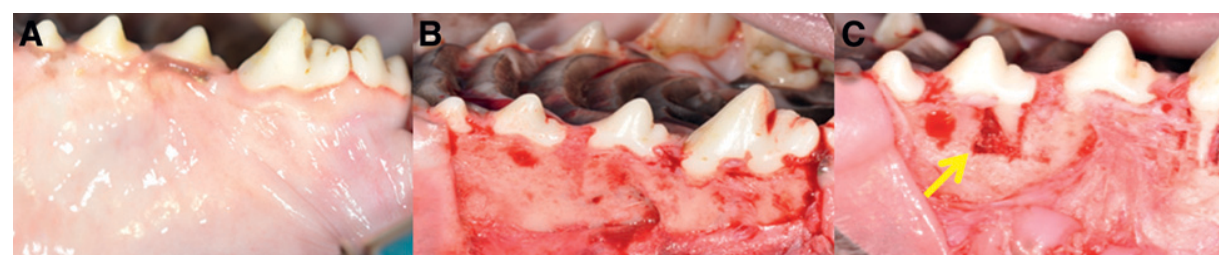

Figure I.

Surgical protocol. A) Mandible segment showing the premolars and molars in a canine model. B) Exposure of bone using a full-thickness flap. C) Creation of a 3-walled bony defect (arrow).

immunosorbent assay (ELISA) using adipocytokines canine kits. . 7 This analysis was quantitative depending on the standard curve of each test for the standard curve of each item.

\section{Periodontal Parameters}

Periodontal parameters $\left(\mathrm{PI},{ }^{4}\right.$ $\mathrm{BOP},{ }^{4}$ and $\mathrm{PD}^{23}$ ) were measured preoperatively and after

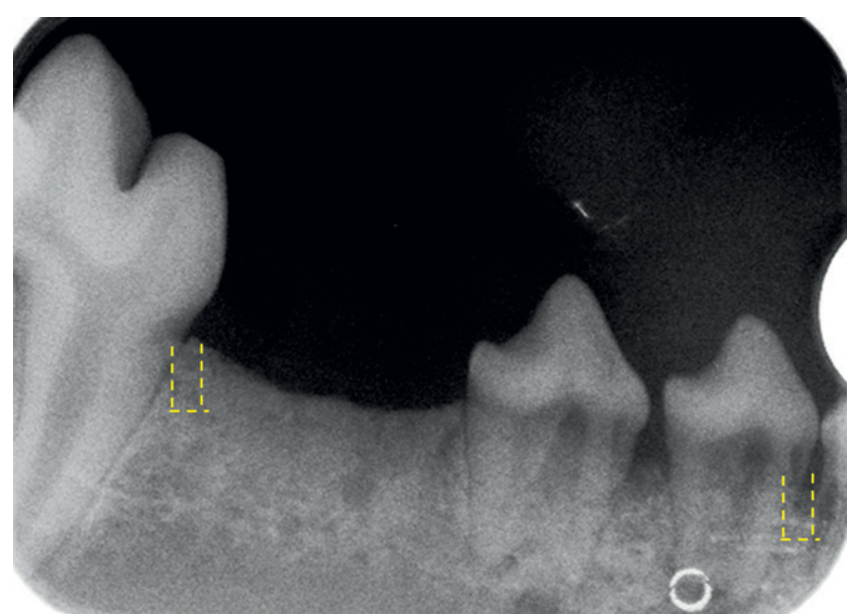

Figure 2.

Creation of 3-walled bony defects (dashed lines) on the mesial aspect of the $P 2$ and $M 1$ teeth in all quadrants.

of NSPT. GCF samples were collected from all quadrants using a no. 30 sterile paper point. The sterile paper point was inserted in the mesial gingival crevice of P2 and M1 of each quadrant and held in place for 60 seconds. After sample collection, paper points were transferred to sterile microcentrifuge $^{\ddagger}$ tubes. The adsorbed volume of GCF in each paper point was weighed using an analytic digital balance, $§ \S$ after which the samples were immediately frozen at $-80^{\circ} \mathrm{C}$ until analysis. To determine the amount of GCF, the adsorbed volume in each single paper point with microcentrifuge tubes was weighed on the analytical balance. The volume of GCF was determined by measuring the difference between the weights of microcentrifuge tubes containing a no. 30 paper point with and without adsorbed GCF. The GCF density volume was considered as 1 , and the difference in weight showed the GCF volume. ${ }^{22}$

Paper points were subjected to centrifugation at 13,000 rpm for 15 minutes in a cold room $\left(4^{\circ} \mathrm{C}\right)$. Each paper point was eluted by $500 \mu \mathrm{L}$ sterilized elution buffer $(0.05 \%$ polysorbate $20 \mathrm{lll}$ in phosphatebuffered saline [pH 7.4]) for 24 hours. Inflammatory markers (IL-6, IL-8, IL-1, IL-1 $\beta$, and TNF- $\alpha$ ) were measured in the eluted solution by enzyme-linked
1, 2, and 3 weeks of NSPT. PD was measured using a graded probe."\# Periodontal parameters were measured on six sites (mesio-buccal, mid-buccal, distobuccal, mesio-palatal/lingual, midpalatal/lingual, and disto-palatal/lingual) around teeth.

\section{Statistical Analyses}

Statistical analysis was performed using a software program. ** Differences in clinical parameters and GCF cytokine concentrations in healthy dogs and dogs with diabetes with experimental periodontitis were assessed using one-way analysis of variance. For multiple comparisons, Bonferroni post hoc test was performed. $P$ values $<0.05$ were considered statistically significant.

\section{RESULTS}

\section{Fasting Blood Glucose Levels}

The mean fasting blood glucose levels were significantly higher in dogs with STZ-induced diabetes $(453 \pm 35.2 \mathrm{mg} / \mathrm{dL})$ compared with those without STZ-induced diabetes $(78.3 \pm 5.4 \mathrm{mg} / \mathrm{dL})(P<0.01)$.

\section{Baseline GCF Cytokine Levels in Dogs With and Without Diabetes}

At baseline, the mean \pm SD IL- 6 levels were significantly higher in dogs with STZ-induced diabetes $(4,506.1 \pm 3,188.8 \mathrm{pg} / \mathrm{mL})$ compared with those without STZ-induced diabetes (1,592 $\pm 2,209.8$ $\mathrm{pg} / \mathrm{mL})(P<0.01)$. The mean IL-8 levels were significantly higher in dogs with STZ-induced diabetes $(96.7 \pm 85.3 \mathrm{pg} / \mathrm{mL})$ compared with those without diabetes $(21.6 \pm 16.7 \mathrm{pg} / \mathrm{mL})(P<0.05)$. There was no statistically significant difference in the mean concentrations of IL- 1 , IL- $1 \beta$, and TNF- $\alpha$ among dogs with and without diabetes (Table 1 ).

\section{Baseline and Postoperative Cytokine Levels in the GCF of Dogs With and Without Diabetes}

In healthy dogs, IL- 1 , IL- 6 , IL- 8 , TNF- $\alpha$, and IL- $1 \beta$ levels were significantly higher at baseline compared

\footnotetext{
邦 Eppendorf, Sigma-Aldrich, St. Louis, MO.

$\S \S$ ABT 120-5DM, Kern and Sohn, Balingen, Germany

Ill Tween 20, Thermo Scientific, Thermo Fisher Scientific, Waltham, MA

II Ascent Multiskan, Thermo Scientific, Waltham, MA.

\#\# Hu-Friedy.

*** SPSS v.18, IBM, Chicago, IL.
} 
Table I.

Preoperative (baseline) Cytokine Levels (mean $\pm \mathrm{SD}$, in $\mathrm{pg} / \mathrm{mL}$ ) in Dogs With Diabetes and Healthy Dogs

\begin{tabular}{lcc}
\hline Cytokines & $\begin{array}{c}\text { Dogs With } \\
\text { STZ-Induced Diabetes }\end{array}$ & $\begin{array}{c}\text { Dogs Without } \\
\text { STZ-Induced Diabetes }\end{array}$ \\
\hline IL-6 & $4,506.1 \pm 3,188.8^{*}$ & $1,592 \pm 2,209.8^{*}$ \\
TNF- $\alpha$ & $64.6 \pm 52.9$ & $41.4 \pm 33.5$ \\
IL-8 & $96.7 \pm 85.3^{\dagger}$ & $21.6 \pm 16.7^{\dagger}$ \\
IL- I $\beta$ & $165.5 \pm 182.7$ & $119.5 \pm 65.8$ \\
IL-I & $76.2 \pm 63.9$ & $64.3 \pm 45.2$ \\
\hline
\end{tabular}

* $P<0.01$.

$\dagger P<0.05$. with those measured at weeks $1 \quad(P<0.01), 2$ $(P<0.05)$, and $3(P<0.01)$ after NSPT. There was no statistically significant difference in the cytokine concentrations (IL- 1 , IL-1 $\beta$, IL- 6 , IL-8, and TNF- $\alpha$ ) among weeks 1,2 , and 3 after NSPT (Table 2).

In dogs with STZ-induced diabetes, there was no statistically significant difference in the mean concentrations of IL- 1 , IL- $1 \beta$, IL- 6 , IL- 8 , and TNF- $\alpha$ at baseline and those measured at week 1 after NSPT (Table 3). IL-1, IL-6, IL-8, TNF- $\alpha$, and IL-1 $\beta$ levels were significantly higher at baseline compared with those measured at weeks 2 and $3(P<0.05)$ after NSPT. IL-1, IL-6, IL-8, TNF- $\alpha$, and IL- $1 \beta$ levels were significantly higher after week 1 of NSPT than those measured at weeks $2(P<0.05)$ and $3(P<0.05)$ after NSPT. There was no statistically significant difference in the mean IL- 1 , IL- 6 , IL- 8 , TNF- $\alpha$, and IL- $1 \beta$ levels measured at weeks 2 and 3 after NSPT (Table 3).

Table 2.

Cytokine Levels (mean $\pm \mathrm{SD}$, in $\mathrm{pg} / \mathrm{mL}$ ) in the GCF of Healthy Dogs at Baseline and at Weeks 1, 2, and 3 After NSPT

\begin{tabular}{lcccc}
\hline Cytokine & Preoperative & I Week After NSPT & 2 Weeks After NSPT & 3 Weeks After NSPT \\
\hline IL-6 & $1,592 \pm 2,209.8^{* \dagger}$ & $1,076.8 \pm 728.7^{*}$ & $1,012.3 \pm 1,209.3^{\dagger}$ & $1,008.2 \pm 1,182.2^{\ddagger}$ \\
TNF- $\alpha$ & $41.4 \pm 33.5^{* \dagger}$ & $21.2 \pm 12.1^{*}$ & $12.5 \pm 9.1^{\dagger}$ & $10.08 \pm 13.6^{\ddagger}$ \\
$\mathrm{IL}-8$ & $21.6 \pm 16.7^{* \dagger}$ & $11.0 \pm 7.4^{*}$ & $8.6 \pm 7.6^{\dagger}$ & $6.2 \pm 9.9^{\ddagger}$ \\
$\mathrm{IL}-1 \beta$ & $119.5 \pm 65.8^{* \dagger}$ & $37.0 \pm 33.8^{*}$ & $11.1 \pm 13.4^{\dagger}$ & $7.05 \pm 8.5^{\ddagger}$ \\
$\mathrm{IL}-1$ & $76.2 \pm 63.9^{* \dagger}$ & $21.7 \pm 11.4^{*}$ & $17.6 \pm 2.6^{\dagger}$ & $7.43 \pm 6.9^{\ddagger}$ \\
\hline
\end{tabular}

* Baseline compared with 1 week after NSPT, $P<0.01$.

$\dagger$ Baseline compared with 2 weeks after NSPT, $P<0.05$

\# Baseline compared with 3 weeks after NSPT, $P<0.01$.

Table 3.

Cytokine Concentrations (mean $\pm \mathrm{SD}$, in $\mathrm{pg} / \mathrm{mL}$ ) in the GCF of Dogs With STZ-Induced Diabetes at Baseline and Weeks 1, 2, and 3 After NSPT

\begin{tabular}{lcccc}
\hline Cytokine & Preoperative & I Week After NSPT & 2 Weeks After NSPT & 3 Weeks After NSPT \\
\hline IL-6 & $4,506.14 \pm 3,188.8^{*}$ & $4,022.42 \pm 1,276.3^{\dagger \S}$ & $1,368.96 \pm 1,085.7^{* \dagger}$ & $1,131.8 \pm 314.7^{\ddagger \S}$ \\
TNF- $\alpha$ & $64.6 \pm 52.9^{*}$ & $31.8 \pm 10.2^{\dagger \S}$ & $7.8 \pm 5.6^{* \dagger}$ & $7.6 \pm 6.9^{\ddagger \S}$ \\
IL-8 & $96.7 \pm 85.3^{* \dagger}$ & $84.2 \pm 77.7^{\dagger \S}$ & $14.6 \pm 7.3^{* \dagger}$ & $8.7 \pm 4.2^{\ddagger \S}$ \\
IL-| $\beta$ & $165.5 \pm 182.7^{* \ddagger}$ & $114.1 \pm 109^{\dagger \S}$ & $20.9 \pm 15.3^{* \dagger}$ & $7.7 \pm 5.7^{\ddagger \S}$ \\
IL-I & $64.3 \pm 45.2^{* \ddagger}$ & $31.9 \pm 14.8^{\dagger \S}$ & $21.1 \pm 25.6^{* \dagger}$ & $7.7 \pm 1^{\ddagger \S}$ \\
\hline
\end{tabular}

* Baseline compared with week 2 after NSPT, $P<0.05$

$\uparrow$ Week 1 after NSPT compared with week 2 after NSPT, $P<0.05$

\# Baseline compared with week 3 after NSPT, $P<0.05$.

$\S$ Week 1 after NSPT compared with week 3 after NSPT, $P<0.05$ 


\section{Periodontal Parameters in Dogs With and Without Diabetes}

The preoperative periodontal parameters were comparable among dogs with and without STZ-induced diabetes. In dogs without STZ-induced diabetes, significant reductions in PI $(P<0.001)$, and BOP $(P<0.001)$, occurred 1 week after NSPT. In dogs with STZ-induced diabetes, significant reductions in PI $(P<0.01)$, BOP $(P<0.01)$, and PD $\geq 3 \mathrm{~mm}$ $(P<0.01)$ occurred 2 weeks after NSPT. After 3 weeks of NSPT, periodontal parameters in dogs with and without STZ-induced diabetes were comparable (Table 4).

\section{DISCUSSION}

A critical factor that adds value to the present results is standardization of the methodology. In the present study, male beagle dogs with comparable ages are used. In addition, a clinical scenario of adult CP was depicted via induction of standardized periodontal osseous defects. Moreover, optimal consistency was also ensured in the methodologies adopted in clinical examination and laboratory-based investigations (ELISA).

It is generally accepted that the severity of periodontal inflammation modulates the GCF cytokine concentration in individuals with and without diabetes.9,10,24 One explanation for this is that the increased formation and accumulation of AGEs in periodontal tissues in a chronic hyperglycemic state increases the intensity of the immunoinflammatory response to periodontal pathogens because inflammatory cells, such as monocytes and macrophages, have receptors for AGEs. ${ }^{25,26}$ Interaction between AGEs and their receptors stimulates the production of proinflammatory proteins. ${ }^{9,26}$ Moreover, it was claimed that ROS (a potential mechanism for accelerated tissue injury) produced as a result of oxidative stress under periodontal inflammatory conditions causes a state of hyperinflammation, thereby enhancing the production of proinflammatory cytokines. ${ }^{16}$ Furthermore, studies ${ }^{10,12}$ also reported comparable IL-1 $\beta$ levels in patients with CP with and without type 2 diabetes. These results suggest that chronic hyperglycemia and CP are two inflammatory conditions that contribute to enhancing the production and accumulation of proinflammatory cytokines in the GCF.

In the present study, GCF concentrations of IL-6 and IL-8 were significantly higher in dogs with STZinduced diabetes compared with dogs without STZinduced diabetes. This may be explained by the possibility that elevated levels of AGEs in the serum and gingival tissues of dogs with STZ-induced diabetes may have caused a state of enhanced oxidative stress, thereby increasing the concentrations of IL- 6 and IL-8 in the GCF. The present results support the study by Engebretson et al., ${ }^{13}$ in which the authors reported GCF IL- 8 levels to be significantly higher in patients with periodontitis with type 2 diabetes compared with medically healthy patients with periodontitis. Therefore, it may be hypothesized that, in patients with periodontitis with well-controlled diabetes, IL- 8 levels are similar to those in medically healthy individuals with periodontitis. Additional studies are warranted in this regard.

NSPT with and without adjunctive remedies (such as antibiotics and phototherapy) is an effective means of plaque control and treating periodontal inflammatory conditions. ${ }^{19,20,27}$ In the present study, baseline periodontal parameters and GCF concentrations of IL-1, IL-1 $\beta$, IL-6, IL-8, and TNF- $\alpha$ are significantly

\section{Table 4.}

\section{Periodontal Parameters at Baseline and After NSPT in Dogs With and Without STZ-Induced Diabetes}

\begin{tabular}{|c|c|c|c|c|c|c|c|c|}
\hline \multirow[b]{2}{*}{ Parameters } & \multicolumn{4}{|c|}{ Dogs Without STZ-Induced Diabetes } & \multicolumn{4}{|c|}{ Dogs With STZ-Induced Diabetes } \\
\hline & Baseline & $\begin{array}{c}\text { After } \\
\text { Week I } \\
\text { of NSPT }\end{array}$ & $\begin{array}{c}\text { After } \\
\text { Week } 2 \\
\text { of NSPT } \\
\end{array}$ & $\begin{array}{c}\text { After } \\
\text { Week } 3 \\
\text { of NSPT } \\
\end{array}$ & Baseline & $\begin{array}{c}\text { After } \\
\text { Week I } \\
\text { of NSPT } \\
\end{array}$ & $\begin{array}{c}\text { After } \\
\text { Week } 2 \\
\text { of NSPT } \\
\end{array}$ & $\begin{array}{c}\text { After } \\
\text { Week } 3 \\
\text { of NSPT } \\
\end{array}$ \\
\hline PI (\% of sites) & $100 * \uparrow キ$ & $40.6^{*}$ & $23.5^{\dagger}$ & $14.6^{\ddagger}$ & 100\|ศ & 55.6 & $28.4 \|$ & $20.1^{\pi}$ \\
\hline BOP (\% of sites) & $100 * \uparrow キ$ & $53.2^{*}$ & $16.6^{\dagger}$ & $10.5^{\ddagger}$ & $100 \| \boldsymbol{\|}$ & 60.1 & $35.8^{\|}$ & 25.5 I \\
\hline $\mathrm{PD} \geq 3 \mathrm{~mm}$ (\% of sites) & $70.5^{\ddagger \S}$ & 45.2 & $16.6 \S$ & $9.5^{\ddagger}$ & $100 \| \boldsymbol{\|}$ & 84.5 & $30.6 \|$ & $18.4 \pi$ \\
\hline
\end{tabular}

* Baseline compared with week 1 after NSPT, $P<0.001$

$\dagger$ Baseline compared with week 2 after NSPT, $P<0.001$.

‡ Baseline compared with week 3 after NSPT, $P<0.01$.

$\S$ Baseline compared with week 2 after NSPT, $P<0.05$.

$\|$ Baseline compared with week 2 after NSPT, $P<0.01$.

Il Baseline compared with week 3 after NSPT, $P<0.01$. 
reduced at 1 week after NSPT in dogs without STZinduced diabetes. However, in dogs with STZinduced diabetes, periodontal parameters and GCF levels of the aforementioned cytokine levels showed the earliest yet significant reductions after 2 weeks of NSPT. From a clinical perspective, these results reflect that, in medically healthy patients, a single therapy of NSPT is effective in reducing periodontal inflammation. However, the beneficial outcomes of NSPT seem to be delayed under immunocompromised conditions (in the present scenario, STZ-induced diabetes). This delay most likely may be associated with the increased accumulation of oxidative stress and AGEs in the periodontal tissues of dogs with STZ-induced diabetes. Nevertheless, the role of other factors, such as regular oral hygiene maintenance regimens and tobacco habits (such as smoking and tobacco chewing), that may influence the outcomes of NSPT cannot be disregarded.

A limitation of the present study is that the results were based on short-term outcomes of NSPT on GCF cytokine profile. Therefore, from a clinical perspective, it is hypothesized that, in patients with a long history of periodontitis and chronic hyperglycemia, periodontal parameters, GCF cytokine profile, and outcomes of NSPT are compromised to a greater extent compared with patients without systemic illnesses. Moreover, it was shown previously that periodontal parameters are comparable in patients with well-controlled diabetes and individuals without diabetes. ${ }^{4}$ Therefore, it is tempting to speculate that patients with periodontitis with well-controlled diabetes demonstrate the same rate of cytokine reduction after NSPT as that observed in medically healthy individuals with periodontitis. Additional investigations, such as assessment of serum cytokine levels and immunohistochemical examinations, are warranted to assess the pathophysiology of periodontitis under chronic hyperglycemic conditions. However, additional studies are warranted to assess this hypothesis.

\section{CONCLUSIONS}

NSPT reduces GCF levels of proinflammatory cytokines in dogs with and without STZ-induced diabetes; however, chronic hyperglycemia seems to retard the effect of NSPT on GCF cytokine concentration.

\section{ACKNOWLEDGMENTS}

The authors thank the College of Dentistry Research Center and Deanship of Scientific Research at King Saud University, Riyadh, Saudi Arabia, for funding this research project (project FR 0125). The authors report no conflicts of interest related to this study.

\section{REFERENCES}

1. Brito F, Almeida S, Figueredo CM, Bregman R, Suassuna $\mathrm{JH}$, Fischer RG. Extent and severity of chronic periodontitis in chronic kidney disease patients. J Periodontal Res 2012;47:426-430.

2. Savioli C, Ribeiro AC, Fabri GM, et al. Persistent periodontal disease hampers anti-tumor necrosis factor treatment response in rheumatoid arthritis. J Clin Rheumatol 2012;18:180-184.

3. Javed F, Ramalingam S, Ahmed HB, et al. Oral manifestations in patients with neurofibromatosis type-1: A comprehensive literature review. Crit Rev Oncol Hematol 2014; pii:S1040-8428(14)00049-3.

4. Javed $F$, Näsström K, Benchimol D, Altamash $M$, Klinge B, Engström PE. Comparison of periodontal and socioeconomic status between subjects with type 2 diabetes mellitus and non-diabetic controls. J Periodontol 2007;78:2112-2119.

5. Javed F, Klingspor L, Sundin U, Altamash M, Klinge B, Engström PE. Periodontal conditions, oral Candida albicans and salivary proteins in type 2 diabetic subjects with emphasis on gender. BMC Oral Health 2009; 9:12.

6. Lin SJ, Tu YK, Tsai SC, Lai SM, Lu HK. Non-surgical periodontal therapy with and without subgingival minocycline administration in patients with poorly controlled type II diabetes: A randomized controlled clinical trial. Clin Oral Investig 2012;16:599-609.

7. Santos VR, Lima JA, Gonçalves TE, et al. Receptor activator of nuclear factor-kappa B ligand/osteoprotegerin ratio in sites of chronic periodontitis of subjects with poorly and well-controlled type 2 diabetes. JPeriodontol 2010;81:1455-1465.

8. Javed F, Al-Askar M, Al-Rasheed A, Babay N, GalindoMoreno P, Al-Hezaimi K. Comparison of self-perceived oral health, periodontal inflammatory conditions and socioeconomic status in individuals with and without prediabetes. Am J Med Sci 2012;344:100-104.

9. Javed F, Al-Askar M, Al-Hezaimi K. Cytokine profile in the gingival crevicular fluid of periodontitis patients with and without type 2 diabetes: A literature review. $J$ Periodontol 2012;83:156-161.

10. Correa FO, Gonçalves D, Figueredo CM, Gustafsson A, Orrico SR. The short-term effectiveness of non-surgical treatment in reducing levels of interleukin-1beta and proteases in gingival crevicular fluid from patients with type 2 diabetes mellitus and chronic periodontitis. J Periodontol 2008;79:2143-2150.

11. Duarte PM, de Oliveira MC, Tambeli CH, Parada CA, Casati MZ, Nociti FH Jr. Overexpression of interleukin1beta and interleukin- 6 may play an important role in periodontal breakdown in type 2 diabetic patients. $J$ Periodontal Res 2007;42:377-381.

12. Navarro-Sanchez AB, Faria-Almeida R, BasconesMartinez A. Effect of non-surgical periodontal therapy on clinical and immunological response and glycaemic control in type 2 diabetic patients with moderate periodontitis. J Clin Periodontol 2007;34:835-843.

13. Engebretson SP, Vossughi F, Hey-Hadavi J, Emingil G, Grbic JT. The influence of diabetes on gingival crevicular fluid beta-glucuronidase and interleukin-8. $J$ Clin Periodontol 2006;33:784-790.

14. Bulut U, Develioglu H, Taner IL, Berker E. Interleukin-1 beta levels in gingival crevicular fluid in type 2 diabetes mellitus and adult periodontitis. J Oral Sci 2001;43: 171-177. 
15. Kurtiş B, Develioğlu H, Taner IL, Baloș K, Tekin IO. IL-6 levels in gingival crevicular fluid (GCF) from patients with non-insulin dependent diabetes mellitus (NIDDM), adult periodontitis and healthy subjects. J Oral Sci 1999; 41:163-167.

16. Wei PF, Ho KY, Ho YP, Wu YM, Yang YH, Tsai CC. The investigation of glutathione peroxidase, lactoferrin, myeloperoxidase and interleukin-1beta in gingival crevicular fluid: Implications for oxidative stress in human periodontal diseases. J Periodontal Res 2004;39:287-293.

17. Takeda M, Ojima M, Yoshioka H, et al. Relationship of serum advanced glycation end products with deterioration of periodontitis in type 2 diabetes patients. $J$ Periodontol 2006; 77:15-20.

18. Jiang ZL, Cui YQ, Gao R, et al. Study of TNF- $\alpha$, IL-1 $\beta$ and LPS levels in the gingival crevicular fluid of a rat model of diabetes mellitus and periodontitis. Dis Markers 2013;34:295-304.

19. Qadri T, Javed F, Poddani P, Tunér J, Gustafsson A. Long-term effects of a single application of a watercooled pulsed Nd:YAG laser in supplement to scaling and root planing in patients with periodontal inflammation. Lasers Med Sci 2011;26:763-766.

20. Qadri T, Poddani P, Javed F, Tunér J, Gustafsson A. A short-term evaluation of Nd:YAG laser as an adjunct to scaling and root planing in the treatment of periodontal inflammation. J Periodontol 2010;81:1161-1166.

21. Anderson HR, Stitt AW, Gardiner TA, Lloyd SJ, Archer DB. Induction of alloxan/streptozotocin diabetes in dogs: A revised experimental technique. Lab Anim 1993;27:281-285.

22. Zhao YB, Meng HX, Chen ZB. The clinical observations and the measurement of gingival crevicular fluid volume during the experimental gingivitis (in Chinese) Zhonghua Kou Qiang Yi Xue Za Zhi 2004;39:42-44.

23. Armitage GC, Svanberg GK, Löe H. Microscopic evaluation of clinical measurements of connective tissue attachment levels. J Clin Periodontol 1977;4:173-190.

24. Javed F, Tenenbaum HC, Nogueira-Filho G, et al. Periodontal inflammatory conditions among gutka chewers and non-chewers with and without prediabetes. $J$ Periodontol 2013;84:1158-1164.

25. Aldridge JP, Lester V, Watts TL, Collins A, Viberti G, Wilson RF. Single-blind studies of the effects of improved periodontal health on metabolic control in type 1 diabetes mellitus. J Clin Periodontol 1995;22:271275.

26. Amir J, Waite M, Tobler J, et al. The role of hyperglycemia in mechanisms of exacerbated inflammatory responses within the oral cavity. Cell Immunol 2011; 272:45-52.

27. de Lima Oliveira AP, de Faveri M, Gursky LC, et al. Effects of periodontal therapy on GCF cytokines in generalized aggressive periodontitis subjects. J Clin Periodontol 2012;39:295-302.

Correspondence: Dr. Khalid Al-Hezaimi, Engineer Abdullah Bugshan Research Chair for Growth Factors and Bone Regeneration, 3D Imaging and Biomechanical Laboratory, College of Applied Medical Sciences and Department of Periodontics and Community Dentistry, King Saud University, Riyadh, Saudi Arabia. E-mail: hezaimik16@gmail.com.

Submitted March 5, 2014; accepted for publication April 27, 2014. 